\title{
Numerical simulation of heat and mass transfer during the absorption of hydrogen in a magnesium hydride
}

\author{
Philippe Marty ${ }^{\mathrm{a}, *}$, J.-F. Fourmigue ${ }^{\mathrm{b}}$, P. De Rango ${ }^{\mathrm{c}, \mathrm{d}}$, \\ D. Fruchart ${ }^{\mathrm{c}, \mathrm{d}}$, J. Charbonnier ${ }^{\mathrm{c}, \mathrm{d}}$ \\ ${ }^{a}$ Laboratoire des Ecoulements Géophysiques et Industriels, BP 53, 38041 Grenoble cedex, France \\ ${ }^{\mathrm{b}}$ CEA-Greth, 17 Rue des Martyrs, 38054 Grenoble cedex 9, France \\ c CRETA, 25 Avenue des Martyrs, BP 166X, 38052 Grenoble cedex, France \\ ${ }^{\mathrm{d}}$ Lab. de Cristallographie, 25 Avenue des Martyrs, BP 166X, 38052 Grenoble cedex, France
}

This paper presents a numerical work aiming at the prediction of the characteristics of an industrial tank filled with hydrides for hydrogen storage. A validation of the method is given and is followed by the resolution of an example which shows the importance of achieving a three-dimensional modelling for the design of an industrial tank. Finally, recent results obtained on a magnesium hydride laboratory tank are given.

Keywords: Hydrogen; Absorption; Heat and mass transfer; Numerical computation; Energy storage; Metal hydride

\section{Introduction}

Beside the problem of performances of a fuel cell, one of the key issues in the development of hydrogen as a future vector of energy is the question of storage of this gas. Several solutions are currently under study:

- The compression technique: for an automotive application, it has been shown (see for instance the DOE recommendations reported in [1]) that a quantity of approximately $5 \mathrm{~kg}$ of hydrogen was required. A simple calculation in a 100 litres tank at ambient temperature indicates that a pressure as high as 700 bar would be necessary. Beside the obvious problems of materials which will arise at such a pressure (mechanical resistance of the walls as well as porosity of stainless steel when exposed to high pressure hydrogen) it has been proved [2] that a huge amount of the hydrogen energy would be consumed in the compression of the gas.

- The liquefaction technique could be used but the need in a highly efficient thermal insulation of the tank walls is a brake to the development of this solution.

\footnotetext{
* Corresponding author. Address: CEA-Greth, 17 Rue des Martyrs, 38054 Grenoble cedex 9, France. Tel.: +33 438783286. E-mail addresses: Philippe.Marty@hmg.inpg.fr, philippe.marty@cea.fr (P. Marty).
} 


\section{Nomenclature}

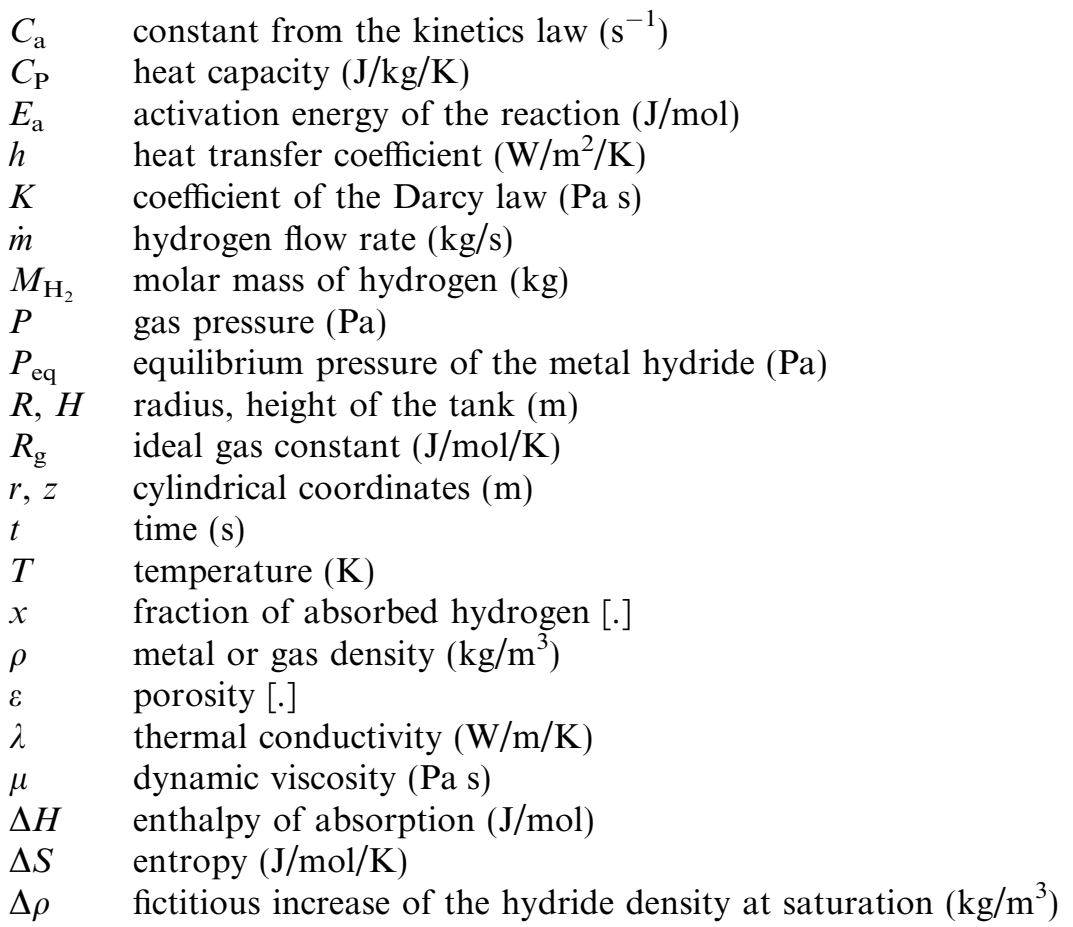

\section{Subscripts}

$\mathrm{m}$, g metal, gas

- The reforming of a liquid fossil fuel is a possibility which obviously has an interest only if the global efficiency of the system reformer/fuel cell has a better efficiency than current engines: this is also a difficult challenge.

- Reversible storage with carbon materials or metallic hydrides is a solution which is currently considered by many car designers for the reason that its application does not involve a highly sophisticated technology. Nevertheless, the constraints are quite severe: the density of a possible candidate should be obviously low, the kinetics should be fast enough to allow a fast filling and a sufficient flow rate to feed the cell in the high power range, the pressure at which absorption occurs at moderate temperature should be high enough, typically a few bars.

This paper concentrates on the last technique which is relative on absorption principle. Magnesium hydride will be considered as a privileged material owing to its high mass performances: recent experiments show that it is able to absorb approximately $5 \%$ of hydrogen in mass [3]. This is higher than carbon-based materials which performances are currently limited to a few per cents [4]. This seems also higher than the performances of new low temperature hydrides reported in [5]. As it will be shown later, the high working temperature of magnesium is a technical difficulty, specially for the feeding of current PEMFC which Nafion membranes cannot work above $70-80^{\circ} \mathrm{C}$. In view of the design of an experience which is under final tests, a numerical approach has been made aiming at predicting and comparing the performances of the tank in terms of time needed to fill in or out the hydrogen content. We shall see that the thermal effects produced by the chemical reaction of absorption may have a critical influence on the time needed to fill the tank. Consequently, it seems obvious that an industrial tank should include an efficient heat exchanger inside the powder contained in the tank walls. From what is known in the development of heat exchangers, it appears that complex geometries 


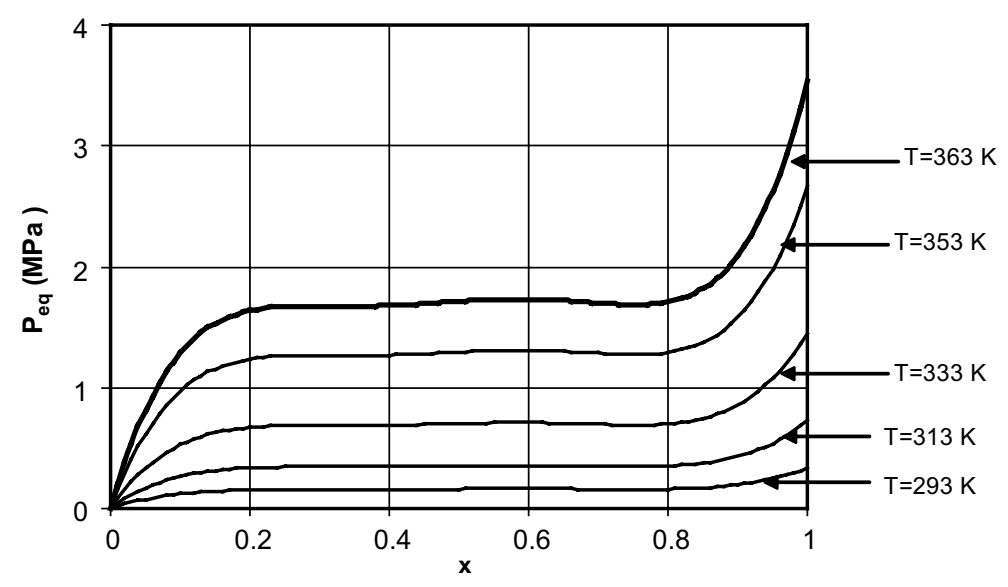

Fig. 1. $P-C-T$ diagram of $\mathrm{LaNi}_{5}$ after Ref. [6].

will soon arise and that only a fully three-dimensional computation will be able to represent the problem. For this reason, we have taken advantage of an already existing industrial code as Fluent for the treatment of the heat and mass transfer which occur during the absorption process. This paper is organised as follows:

- In the first part, a validation of the numerical tool will be discussed by comparing our computer results to those already published in the literature on a $\mathrm{LaNi}_{5}$ tank.

- In the next section, we show how much a 3D geometry influences the result: this is done through the calculation of a tank containing $100 \mathrm{~kg}$ of $\mathrm{LaNi}_{5}$ and then able to store a quantity of hydrogen greater than $1 \mathrm{~kg}$.

- In the last section, we present the experimental magnesium prototype which is under test at CRETA and apply our numerical strategy to this experiment.

\section{Description of the numerical technique}

A typical behaviour of the phenomena occurring during the filling a hydride tank can be described as follows:

- A metallic container, often having stainless steel walls, is filled with a suitably-prepared powder of metal. At starting time $t=0$, the powder is often free from hydrogen and is set at an initial reasonable temperature. The tank is connected via a valve to an hydrogen source having a constant pressure.

- The valve is opened, hydrogen enters the tank and flows through the porous metallic medium. The absorption process starts throughout the powder. Owing to the exothermic character of the absorption process, a violent temperature increase happens everywhere in the tank.

- The equilibrium pressure $P_{\text {eq }}$ of the metal rises as it is an increasing function of the temperature: this is shown in Fig. 1 which shows the dependence of $P_{\text {eq }}$ with the hydrogenation fraction ${ }^{1} x$ for different temperatures. For each value of $T$, a plateau is observed at a pressure in agreement with the Van't Hoff law.

- Although the pressure $P$ in the tank stays rather constant as it is imposed by the external source, the kinetics, i.e. the rate at which hydrogen is absorbed, decreases. As a matter of fact, the experimental analysis shows that the kinetics of hydrides can be suitably fitted by expressions proportional to $\operatorname{Ln} \frac{P}{P_{\mathrm{eq}}}$ or to the difference $P-P_{\text {eq }}$. This explains why, after a short time interval, the reaction almost stops and waits for the

\footnotetext{
${ }^{1}$ The hydrogenation fraction, $x$, is a local quantity which is obtained by dividing the mass of hydrogen which has been absorbed by the mass which is absorbed at saturation.
} 


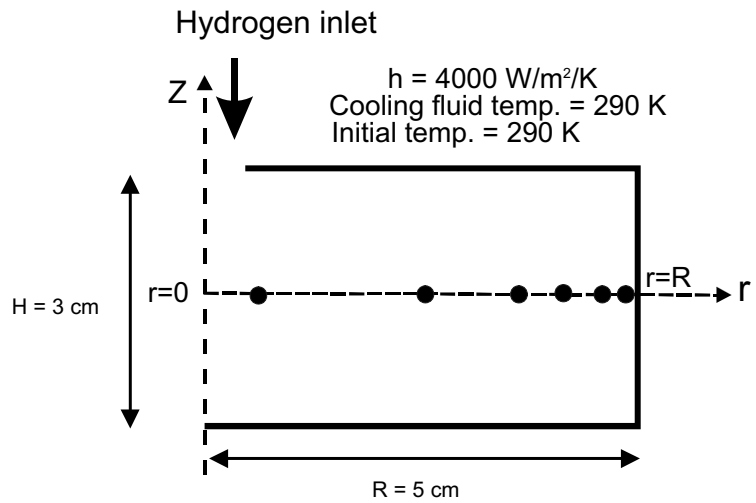

Fig. 2. Sketch of the geometry studied in [7] which has been used as a test case. The circles indicate points where the temperature has been recorded (see Fig. 4).

temperature decrease to proceed further. The essential role that can have an efficient heat transfer is then clear.

The problem which has been used for our numerical test has been published in [7]. The finite volume technique implemented in the commercial code Fluent has been used. The geometry is shown in Fig. 2: it consists in a small axially symmetric tank filled with $\mathrm{LaNi}_{5}$ and having a porosity $\varepsilon=0.5$. Its walls are submitted to a convective heat exchange (heat transfer coefficient $h=4000 \mathrm{~W} / \mathrm{m}^{2} / \mathrm{K}$ and $T=290 \mathrm{~K}$ for the fluid at infinity). This imposes the normal derivative of the temperature at the wall such as $-\lambda \frac{\partial T}{\partial n}=h\left(T-T_{\infty}\right)$ where $n$ stands for the normal direction to the wall. A constant pressure equal to $10^{6} \mathrm{~Pa}$ is imposed at the tank inlet whereas other boundaries receive the classical no-slip $(\vec{V}=0)$ or axis condition $\left(\frac{\partial \vec{V}}{\partial r}=\frac{\partial T}{\partial r}=0\right)$ in $r=0$. The initial temperature is $290 \mathrm{~K}$.

The absorption rate ( $\mathrm{kg}$ of $\mathrm{H}_{2}$ absorbed per unit of time and of volume of porous medium) is defined in [7] as

$$
C_{\mathrm{a}} \mathrm{e}^{-\frac{E_{\mathrm{a}}}{R T}} \cdot \Delta \rho \cdot(1-x) \cdot \operatorname{Ln} \frac{P}{P_{\mathrm{eq}}},
$$

where $C_{\mathrm{a}}=59.187 \mathrm{~s}^{-1}$ is a constant, $E_{\mathrm{a}}=21179 \mathrm{~J} / \mathrm{mol}$ the activation energy and $R_{\mathrm{g}}=8.32 \mathrm{~J} / \mathrm{mol} / \mathrm{K}$ the ideal gas constant. $P$ stands for the absolute pressure and $P_{\text {eq }}$ is the equilibrium pressure as defined in Fig. 1 for example. The quantity $P_{\text {eq }}$ is a function of $x$ and $T$ which is analytically computed from a fifth-order polynomial with respect to $x$. The quantity $\Delta \rho$ is the fictitious increase of density of the metal owing to the hydrogenation process: typically, its value is $54 \mathrm{~kg} / \mathrm{m}^{3}$ for $\mathrm{LaNi}_{5}$. Using the hydrogenation fraction $x$ (already defined and also shown in Fig. 1) allows to express the hydrogenation rate in a formula which is equivalent to Eq. (1):

$$
\frac{\mathrm{d} x}{\mathrm{~d} t}=\frac{C_{\mathrm{a}}}{(1-\varepsilon)}(1-x) \mathrm{e}^{-\frac{E_{\mathrm{a}}}{R_{\mathrm{g}} T}} \operatorname{Ln} \frac{P}{P_{\mathrm{eq}}} .
$$

Momentum equations are then solved in the porous medium using the classical Darcy law:

$$
\vec{\nabla} P=-\frac{\mu}{K} \vec{V}
$$

where the coefficient $K$ is a function of the particle size of the powder.

The continuity equation includes a sink term to take hydrogen absorption into account. Per unit of volume $\left(\mathrm{kg} / \mathrm{m}^{3} / \mathrm{s}\right)$ this equation becomes

$$
\varepsilon \frac{\partial \rho_{\mathrm{g}}}{\partial t}+\operatorname{div}\left(\rho_{\mathrm{g}} \vec{V}\right)=\Delta \rho \cdot(1-\varepsilon) \frac{\mathrm{d} x}{\mathrm{~d} t},
$$


where the gas density $\rho_{\mathrm{g}}$ is deduced from the perfect gas model: $\rho_{\mathrm{g}}=\frac{P \cdot M_{\mathrm{H}_{2}}}{R_{\mathrm{g}} T}$ and where the right-hand side term stands for the hydrogen consumption during the hydrogenation.

The heat equation is

$$
\rho C_{\mathrm{P}} \frac{\partial T}{\partial t}+\left(\rho C_{\mathrm{P}}\right)_{\mathrm{g}} \vec{V} \cdot \vec{\nabla} T=\lambda \nabla^{2} T+\frac{\Delta \rho \cdot(1-\varepsilon) \Delta H}{M_{\mathrm{H}_{2}}} \frac{\mathrm{d} x}{\mathrm{~d} t},
$$

where

$$
\rho C_{\mathrm{P}}=\varepsilon\left(\rho C_{\mathrm{P}}\right)_{\mathrm{g}}+(1-\varepsilon)\left(\rho C_{\mathrm{P}}\right)_{\mathrm{m}}
$$

and

$$
\lambda=\varepsilon \lambda_{\mathrm{g}}+(1-\varepsilon) \lambda_{\mathrm{m}}
$$

are mean values obtained from the classical averaging operation on the metal and the gas. The source term at the right side of Eq. (5) represents the release of heat during the absorption process $\left(\mathrm{W} / \mathrm{m}^{3}\right)$. The porosity $\varepsilon$ is equal to 0.5 and the enthalpy of absorption is $\Delta H=3 \times 10^{4} \mathrm{~J} / \mathrm{mol}$. The conductivity of the pure metal is $2.4 \mathrm{~W} \mathrm{~m}^{-1} \mathrm{~K}^{-1}$ leading, from Eq. (7) to an effective conductivity of the powder approximately equal to $1.2 \mathrm{~W} \mathrm{~m}^{-1} \mathrm{~K}^{-1}$.

Fig. 3 shows the time evolution of the amount of hydrogen which has been stored. Our results are reasonably comparable to those published in [7]. Fig. 4 shows details on the time evolution of the temperature in

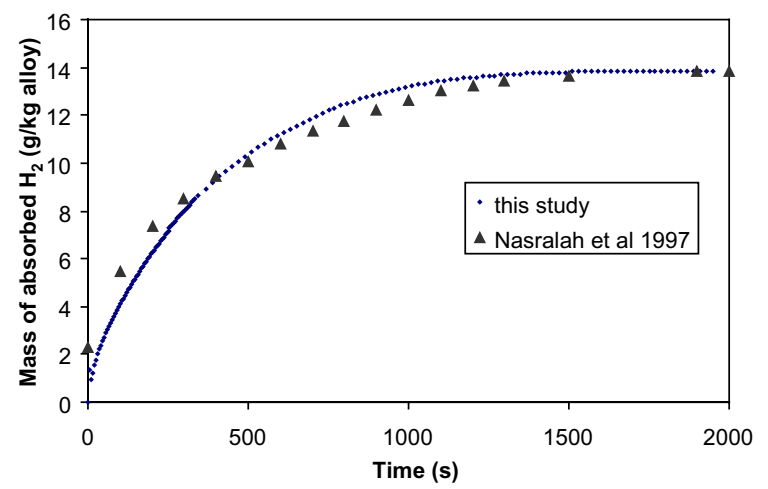

Fig. 3. Time evolution of the mass of absorbed $\mathrm{H}_{2}$ : comparison between our results and those described in [7].

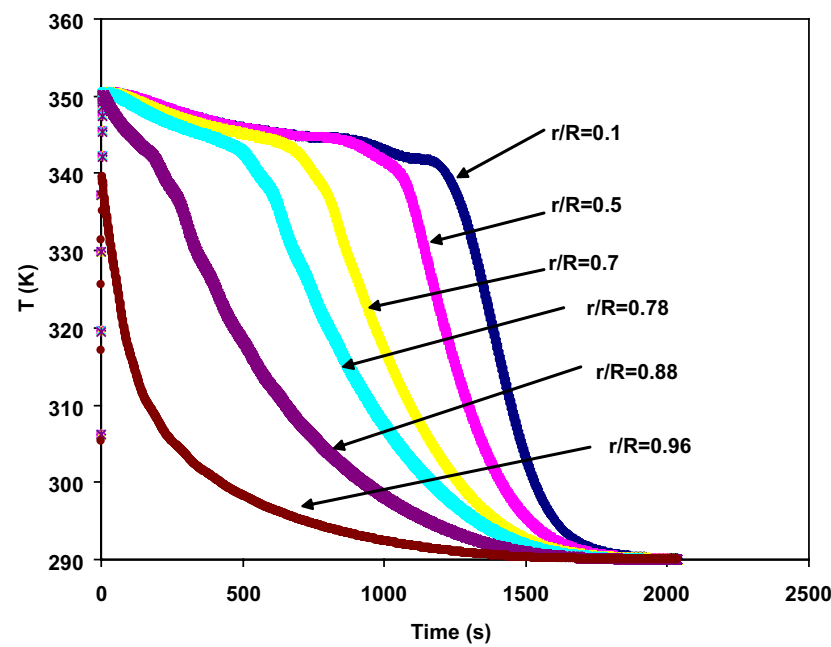

Fig. 4. Time history of the temperature at $r / R=0.1,0.5,0.7,0.78,0.88$ and 0.96 and $z=H / 2$. 
points located at mid-height but at various radii: a global heating of the core of the tank appears whereas the metal near the boundaries experiences an efficient cooling which produces a faster hydriding. The temperature near the axis $(r / R=0.1)$ decreases with a larger time scale than near the cooled wall $(r / R=0.96)$.

The agreement between our results and Ref. [7] is globally good. Uncertainties remain which can be the consequence of various variables which were not explicitly specified in their paper.

\section{3D vs. $2 \mathrm{D}$ calculations}

The above section has shown that the numerical technique was able to describe heat and mass transfer in a hydride tank. The purpose of this section is to show how much a three-dimensional tank geometry is necessary to decrease the time needed to fill a $100 \mathrm{~kg} \mathrm{LaNi}_{5}$ tank. The mass of metal has been chosen such as it is able to

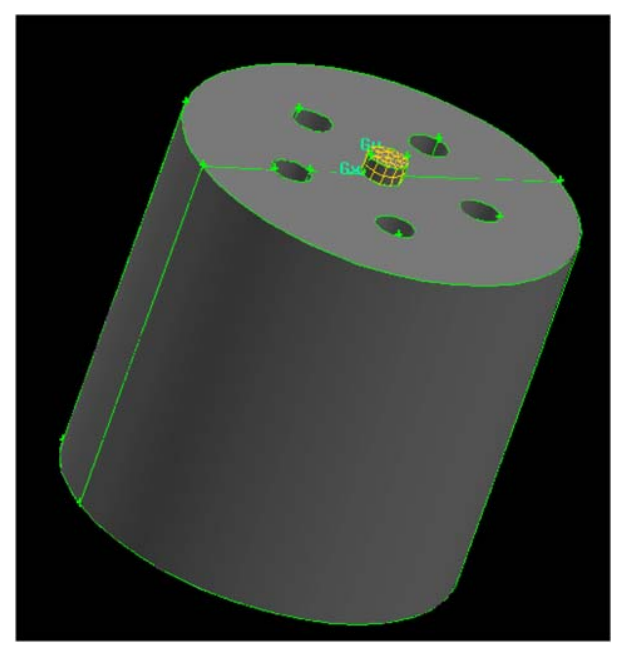

Fig. 5. Tank used for the 2D/3D comparison. Hydrogen is allowed to enter the tank through the central hole. Five axial channels located on the same radius eventually allow the circulation of a coolant.

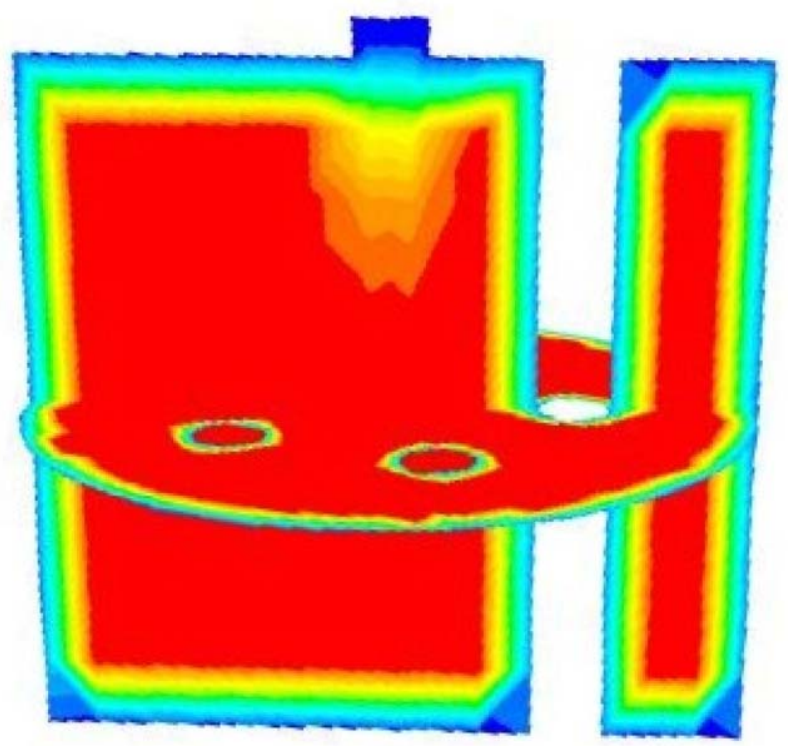

Fig. 6. Temperature field in the tank at time $t=0.5 \mathrm{~s}$ (the whole core of the tank has already reached $245 \mathrm{~K}$ whereas the wall boundaries remain at $240 \mathrm{~K}$ as prescribed by the boundary conditions). 
store a reasonable amount of hydrogen for a car application. Here, $100 \mathrm{~kg}$ of $\mathrm{LaNi}_{5}$ are able to store $1.38 \mathrm{~kg}$ of hydrogen, what is still much less than the $5 \mathrm{~kg}$ required by the DOE recommendation but is nevertheless significant.

Fig. 5 shows a cylindrical tank containing $100 \mathrm{~kg}$ of $\mathrm{LaNi}_{5}$. Its dimensions are $R=20 \mathrm{~cm}$ and $H=38.4 \mathrm{~cm}$ corresponding to a volume of 481 . Porosity $\varepsilon$ is 0.5 . Hydrogen is allowed to enter through the central orifice under a constant pressure of 10 bar. The side, top and bottom walls of the tank are maintained at a constant temperature of $240 \mathrm{~K}$. The purpose of this test is to show much the filing time of the tank is reduced when an internal cooling is provided. This is done thanks to five channels drilled in the axial direction at a constant radius. A coolant maintains the walls of these channels to $240 \mathrm{~K}$, i.e. at the same temperature as the external walls. Without the presence of these five channels, the geometry is axially symmetric and can be modelled with a 2D numerical mesh. On the other hand, adding the presence of these channels renders the geometry fully 3D by breaking the axial symmetry. Fig. 6 shows the temperature field after $0.5 \mathrm{~s}$. The bulk of the tank has an almost constant temperature whereas thermal boundary layers can be seen along the walls of the tank and along the five cooling channels. The hydrogen cold flow inlet is also visible at the top of the figure on the $\mathrm{O}_{z}$-axis. Fig. 7 shows the time trajectory of a particular point in the $\left(P_{\mathrm{eq}}, x\right)$ diagram. This point is located

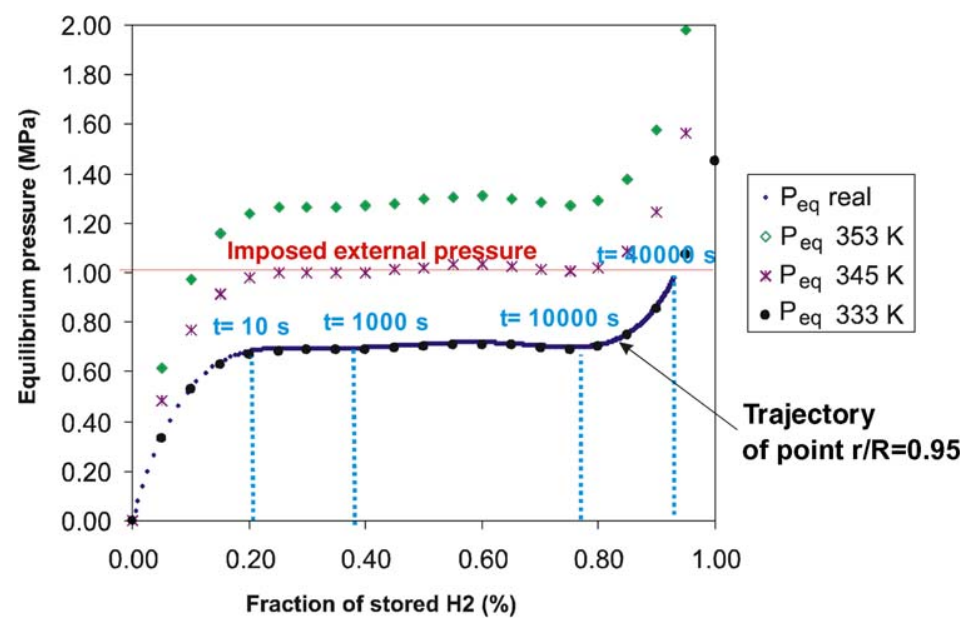

Fig. 7. Effective trajectory of a point located in $r / R=0.95$ in the $P-C-T$ diagram.

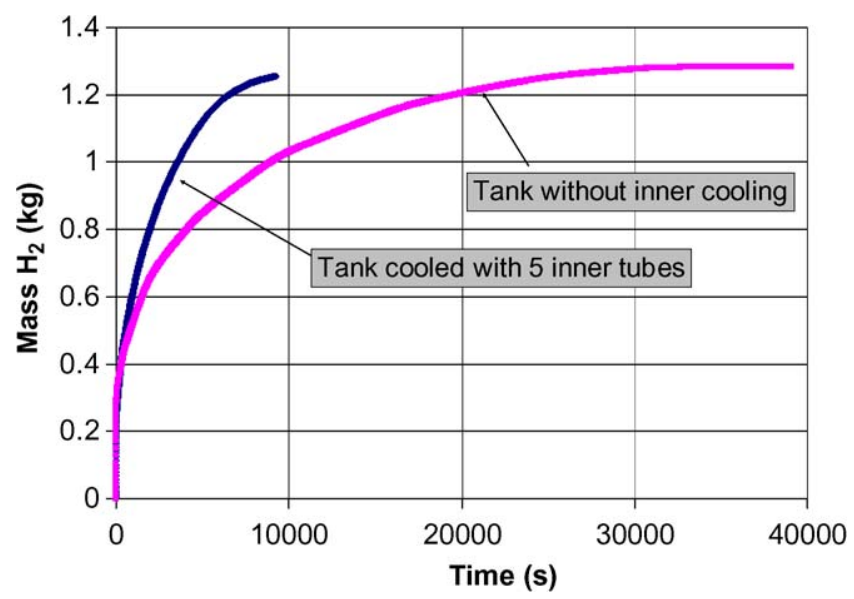

Fig. 8. Time evolution of the stored mass of hydrogen in a $100 \mathrm{~kg} \mathrm{LaNi}_{5}$ tank. The time needed without internal cooling is approximately 3 times larger than with an inner cooling. 
at mid-height and in $r / R=0.95$, i.e. very close from the side wall. We see a plateau around $P_{\text {eq }}=7$ bar which lasts from approximately $t=10 \mathrm{~s}$ to $t=10000 \mathrm{~s}$. The interest of providing an internal cooling appears in Fig. 8 which shows the time evolution of the mass of hydrogen which has been absorbed with or without the cooling. The asymptotic value of both curves is $1.38 \mathrm{~kg}$ as expected. The time $t_{90}$ at which $90 \%$ of the amount of hydrogen has been stored (i.e., $1.24 \mathrm{~kg}$ ) is $8179 \mathrm{~s}$ with the cooling and $23044 \mathrm{~s}$ without the cooling. In this extremely simplified example the filling time has already been reduced by a factor 3 approximately.

\section{Preliminary design of a magnesium experiment}

Fig. 9 shows the experimental tank which consists of a cylindrical stainless steel shell with an inner copper cylinder aimed at cooling the magnesium during the absorption stage.

The kinetics law has been obtained from preliminary isothermal experiments done on a small sample: it has been shown that the hydriding fraction can be fitted by $x=1-(1-\sqrt{k t})^{3}$, where $k=k_{0} \cdot \frac{P-P_{\mathrm{eq}}}{P_{\mathrm{eq}}} \mathrm{e}^{-E_{\mathrm{a}} / R_{\mathrm{g}} T}$. In this expression, $E_{\mathrm{a}}$ is the activation energy of the reaction, $k_{0}$ is a constant and the magnesium equilibrium pressure $P_{\text {eq }}$ is such as

$$
P_{\text {eq }}=10^{5} \exp \left(\frac{\Delta H}{R_{\mathrm{g}} T}-\frac{\Delta S}{R_{\mathrm{g}}}\right)
$$

The evolution of the equilibrium pressure $P_{\text {eq }}$ with temperature is shown in Fig. 10 and Table 1 shows the different variables used in the computation.

The absorption velocity of magnesium is deduced from the experiments: it is a function of $P_{\text {eq }}, P, T$ and $x$ and can be written

$$
\frac{\mathrm{d} x}{\mathrm{~d} t}=\frac{3 k}{2} \frac{(1-x)^{2 / 3}}{1-(1-x)^{1 / 3}} .
$$

It is plotted in Fig. 11 as a function of $T$ for various values of $x$. We see that a too cold temperature (typically less than $400 \mathrm{~K}$ ) stops the reaction. The same tendency is observed for $T$ larger than $650 \mathrm{~K}$. This is because of the equilibrium pressure which reaches 10 bar at this temperature: this makes $k$ vanishing and cancels the velocity of the reaction. Finally, we see that the reaction velocity will be maximum around $620 \mathrm{~K}$ whatever the value of $x$.

Fig. 12 shows the mesh and Fig. 13 shows the velocity streamlines at $t=204 \mathrm{~s}$. Most of the hydrogen flows inside the annular copper ring whereas one part flows radially between the top wall and the copper ring. Fig. 14 shows the time evolution of the total mass flow rate at the inlet. After a very intense peak around

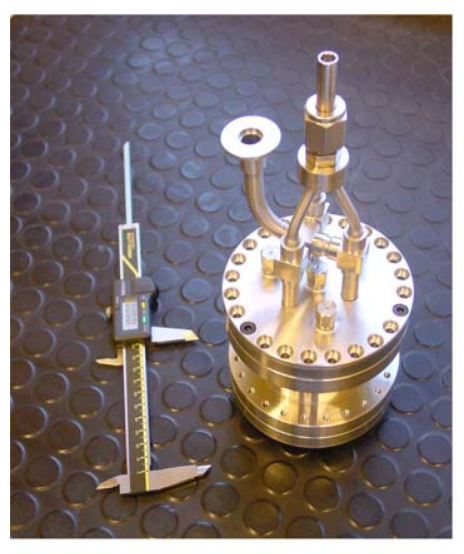

(a)

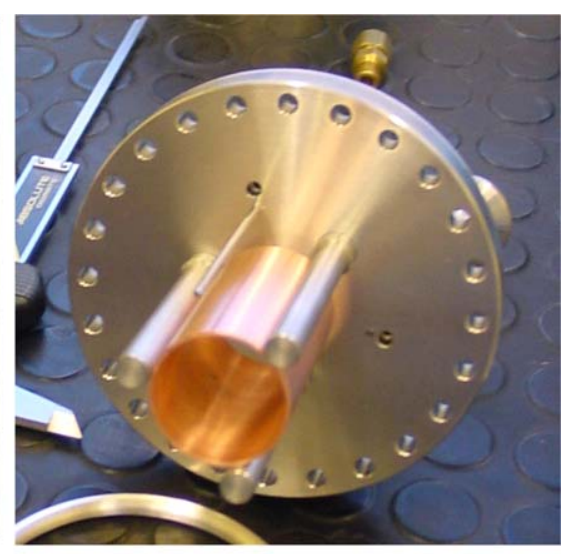

(b)

Fig. 9. Experimental magnesium tank. (a) View of the cooling circuit (b) inner copper ring on which the cooling circuit has been brazed. 


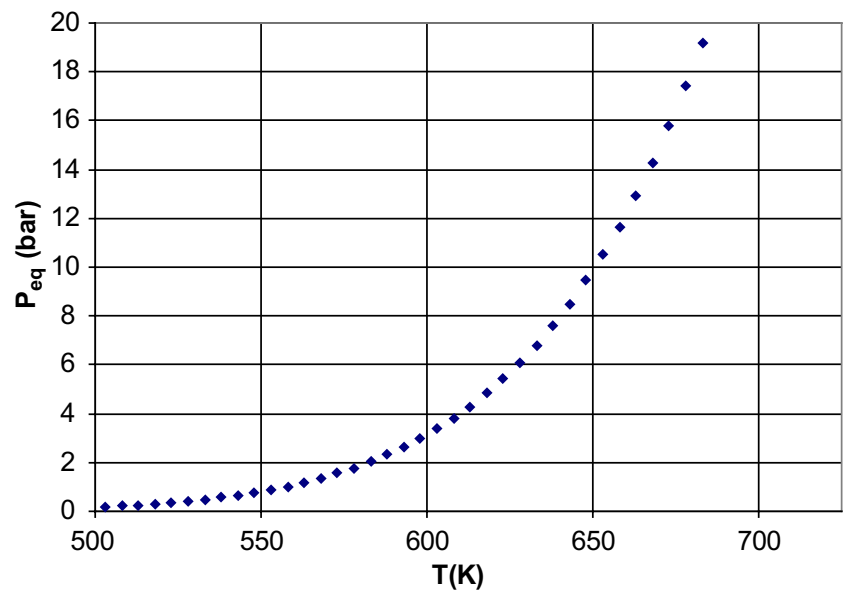

Fig. 10. Dependence of the equilibrium pressure of magnesium with temperature.

Table 1

Numerical values of the variables used in the computation

Height of the tank

Radius of the tank

Radius of the copper ring

Height of the copper ring

Density of magnesium

Porosity

Thermal conductivity of magnesium

Thermal conductivity of hydrogen

Heat capacity of magnesium

Heat capacity of hydrogen

Initial temperature

Hydrogen pressure

Thermal conditions at the external wall

Thermal condition at the copper ring

Temperature of $\mathrm{H}_{2}$ at inlet

Diameter of $\mathrm{H}_{2}$ inlet

$\Delta H$

$\Delta S$

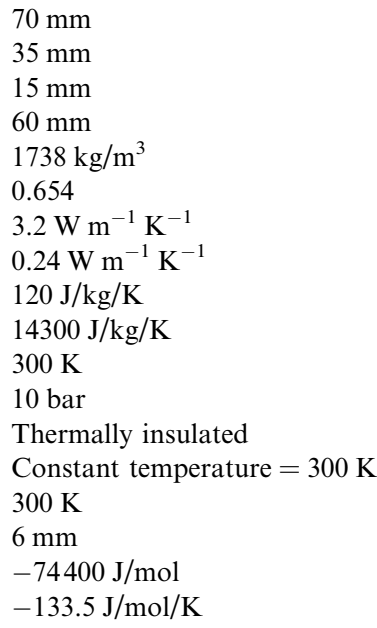

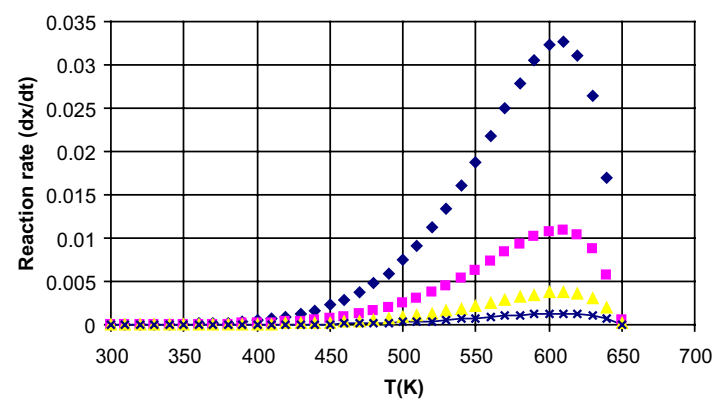

reaction-velocity $x=0.1$
reaction-velocity $x=0.25$
reaction-velocity $x=0.5$
*
reaction-velocity $x=0.75$

Fig. 11. Reaction rate vs. temperature for various values of the hydriding fraction $x$ (pressure $=10$ bar).

$t=0.1 \mathrm{~s}$, this flow rate monotonously decreases. Fig. 15 shows the temperature distribution at $t=13.2 \mathrm{~s}$ : the cold part at $300 \mathrm{~K}$ near $(r=0 ; z=0)$ is a consequence of the inlet of cold hydrogen. The rest of magnesium powder is blocked at $650 \mathrm{~K}$ which is the temperature at which $P_{\text {eq }}=10$ bar. A very thin axially symmetric 


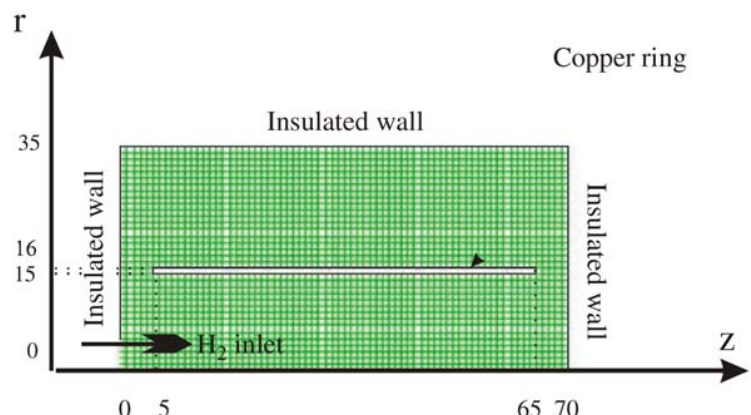

Fig. 12. 2D mesh of the magnesium tank with account for the inner copper ring (dimensions in mm).

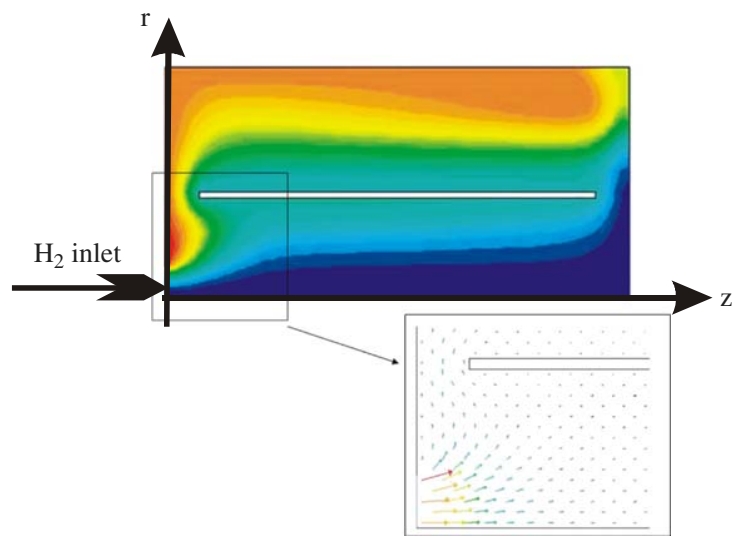

Fig. 13. Velocity streamlines at $t=204 \mathrm{~s}$.

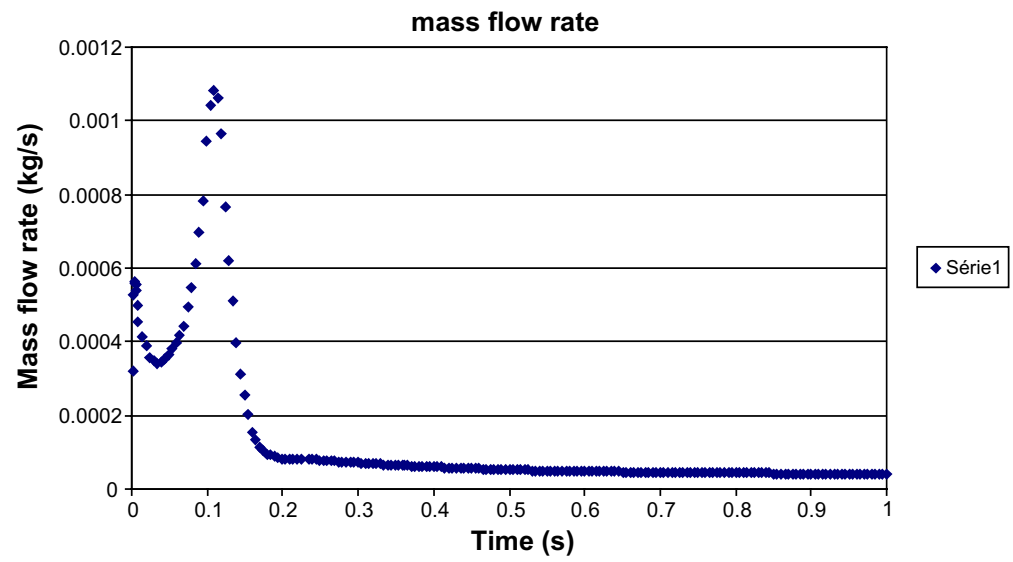

Fig. 14. Time evolution of the mass flow rate during the first second.

front separates these two regions. Fig. 16 shows the time evolution of the mean temperature integrated on the whole volume of the tank. Again, a long plateau is observed until $t=600 \mathrm{~s}$. Fig. 17 shows the contours of the hydriding fraction $x$. The value of $x$ is small except near the above-mentioned front. This is in agreement with Fig. 11. As a matter of fact, the temperature is either too low or too high on both sides of this front and the reaction does not proceed fast enough. 


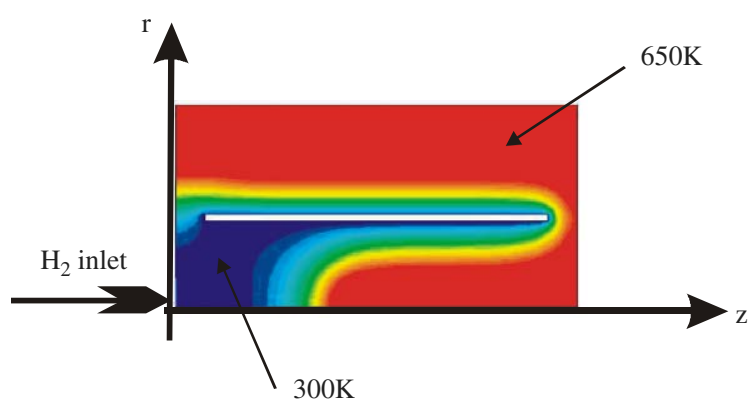

Fig. 15. Temperature contours at time $t=13.2 \mathrm{~s}$.

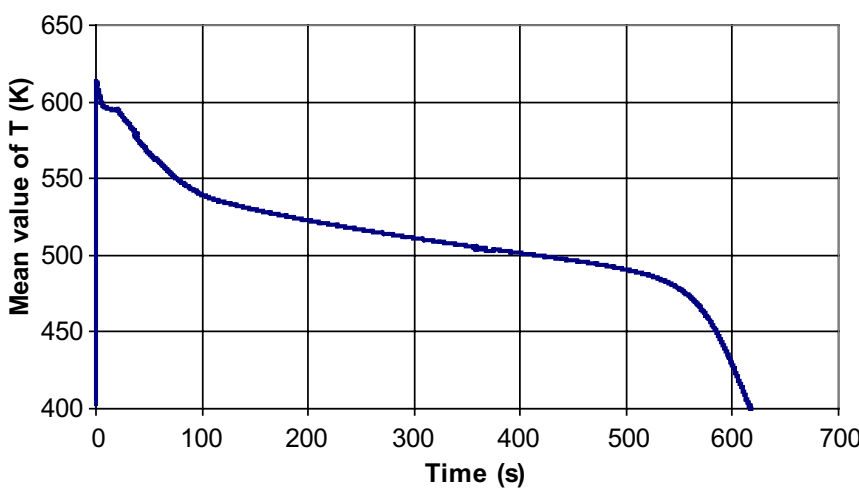

Fig. 16. Time evolution of the mean temperature in the tank.

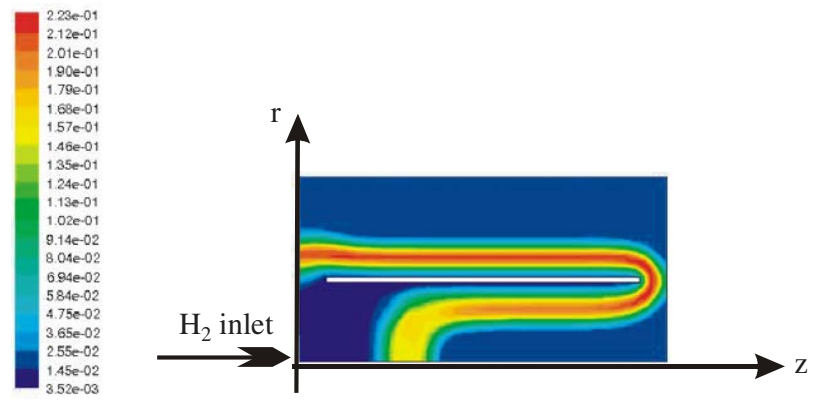

Fig. 17. Hydriding fraction contours at time $t=11.1 \mathrm{~s}$.

Finally, Fig. 18 shows the time evolution of $x$; it confirms that the reaction stops at $t=600 \mathrm{~s}$ although $x$ is only $60 \%$. This is a consequence of the too cold temperature imposed by the copper ring cooling circuit. It is clear that stopping the coolant circulation would allow the reaction to complete until $x=1$.

\section{Conclusions}

A numerical approach has been used for the prediction of heat and mass transfer in an hydride tank during its absorption of hydrogen. The following conclusions can be given: 


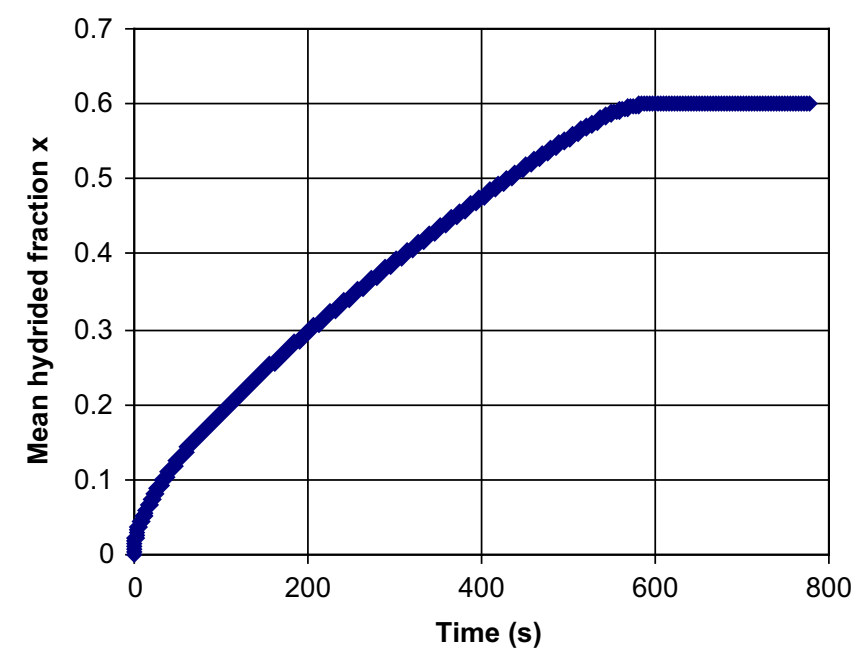

Fig. 18. Time evolution of the mean hydriding fraction.

- Although most of the work described in this paper has been done in 2D geometry, it has been shown that the design of an industrial tank will need the use of a 3D modelling. In this perspective, the use of a commercial code would avoid developing its own tools.

- The CPU time which is required by the computation can be large. Although not discussed in this paper, the time step to reach convergence can be very low, especially at the beginning of the computation when the flow rate reaches its maximum.

- In order to develop an efficient cooling, it is foreseeable that the cylindrical axisymmetric geometry, although simple, could prevent from an intense cooling. Probably the well known plate heat exchanger technology will be of interest.

- The knowledge of the physical properties of the materials will need additional researches. For example, the thermal conductivity of a powder bed will have to be investigated in more details. Along the same line, the effect of dilatation (compaction) of the powder bed during the absorption (desorption) should be taken into account.

\section{Acknowledgments}

CNRS support is acknowledged for financial support through its Energy Research Program. Fabrice Mathey, from Fluent France, is thanked for useful discussions about the absorption model used in this work.

\section{References}

[1] Berry DG, Aceves MS. On-board storage alternatives for hydrogen vehicles. Energy Fuels 1998;12:49.

[2] Bossel U, Eliasson B, Taylor G. The future of hydrogen economy: bright or bleak? Available from: http://www.efcf.com/reports.

[3] Charbonnier J, De Rango P, Fruchart D, Miraglia S, Pontonnier L, Rivoirard S, et al. Hydrogenation of transition element additives (Ti, Nb, Hf) during ball-milling of magnesium hydride. J. Alloys Compd 2004;383:205-8.

[4] Delahaye A, Pentchev I, Aoufi A, Gicquel A. Improvement of hydrogen storage by absorption using 2D modeling of heat effects. AIChE J 2002;48(9):2061-73.

[5] Challet S. Stockage de l'hydrogène dans les hydrures métalliques, thèse de l'Université Paris XII; 2005.

[6] Jemni A, Ben Nasralah S. Study of two-dimensional heat and mass transfer during absorption in a metal-hydrogen reactor. Int $\mathbf{J}$ Hydrogen Energy 1995;20(1):43-52.

[7] Ben Nasralah S, Jemni A. Heat and mass transfer models in metal-hydrogen reactor. Int J Hydrogen Energy 1997;22(1):67-76. 